\title{
Comparison of Three Types of Mesenchymal Stem Cells (Bone Marrow, Adipose Tissue, and Umbilical Cord-Derived) as Potential Sources for Inner Ear Regeneration
}

\author{
Mohammad-Reza Mahmoudian-sani ${ }^{1,2}$ \\ Ameneh Mehri-Ghahfarrokhi² \\ Morteza Hashemzadeh-Chaleshtori ${ }^{1}$ \\ Masoud Saidijam ${ }^{1}$ \\ Mohammad-Saeid Jami
}

\begin{abstract}
In this review, we compared the potential of mesenchymal stem cells derived from bone marrow, adipose tissue and umbilical cord as suitable sources for regeneration of inner ear hair cells and auditory neurons. Our intensive literature search indicates that stem cells in some of adult mammalian tissues, such as bone marrow, can generate new cells under physiological and pathological conditions. Among various types of stem cells, bone marrow-derived mesenchymal stem cells are one of the most promising candidates for cell replacement therapy. Mesenchymal stem cells have been reported to invade the damaged area, contribute to the structural reorganization of the damaged cochlea and improve incomplete hearing recovery. We suggest that bone marrow-derived mesenchymal stem cells would be more beneficial than other mesenchymal stem cells.
\end{abstract}

Keywords: mesenchymal stem cells, hearing loss, regenerating inner ear, hair cell.

${ }^{1}$ Department of Genetics and Molecular Medicine, Hamadan University of Medical Sciences, Hamadan, Iran

${ }^{2}$ Cellular and Molecular Research Center, Basic Health Sciences Institute, Shahrekord University of Medical Sciences, Shahrekord, Iran

Send correspondence to:

Mohammad-Saeid Jami

Cellular and Molecular Research Center, Basic Health Sciences Institute, Shahrekord University of Medical Sciences, Shahrekord, Iran, E-mail: molecular_

hearingloss10025@yahoo.com

Paper submitted to the ITJ-EM (Editorial Manager System) on October 05, 2017;

and accepted on October 27, 2017. 


\section{INTRODUCTION}

Hearing loss is one of the most prevalent disabilities. Approximately over $5 \%$ of the world's population-360 million people-suffer from disabling hearing loss (328 million adults and 32 million children). Hair cells and spiral ganglion neurons are usually damaged in most people with hearing loss. Hearing loss is also the most common sensory disorder in humans. Recent developments in stem cell technology have offered new opportunities to treat deafness. The present review seeks to investigate different types of mesenchymal stem cells (MSCs) (derived from bone marrow, adipose tissue, and umbilical cord) as potential sources for regeneration of inner ear cells. Since cells with stem/progenitor properties appear to be no longer present in the mammalian cochlea three weeks after birth ${ }^{1,2}$, this work mainly focuses on the utilization of exogenous cells and their differentiation into the missing auditory cells. A wide range of stem/ multipotent progenitor cells have been tested for their capability to differentiate toward inner ear sensory cells in vitro and in vivo ${ }^{3-5}$. Several groups have shown the possibility of mouse embryonic stem cells (mESCs) to differentiate into hair cells ${ }^{6,7}$ and auditory neuron-like cells $^{8-11}$. Differentiation methods to induce hair cell-like cells from ESCs or induced pluripotent stem cells (iPSCs) seem to be effective but require great proficiency ${ }^{6,7}$. The beneficial phenotypic plasticity of ESCs is always challenged by ethical questions on derivation and use of these human cells and other concerns such as the likelihood of immune reactions and tumor formation ${ }^{12-14}$.

Alternative sources are mesenchymal tissues. Indeed, inner ear hair cells have been successfully generated in vitro by a mesenchymal-to-epithelial transition ${ }^{15}$. Considering the potential of MSCs to differentiate into neural type cells, these cells would be good candidates for regenerative cell-based therapies ${ }^{16-20}$. Multipotent MSCs are capable to restore damaged mesenchyme via differentiation into mature cells of bone, cartilage, muscle, fat or fibrous tissues. MSCs exhibit remarkable self-renewal capacity and the ability to differentiate not only into osteoblasts, chondrocytes, adipocytes, myocytes, but even also into neurons in vitro and in vivo ${ }^{21}$ and therefore, MSCs have been successfully used in otorhinolaryngology. MSCs produce bioactive anti-inflammatory agents and support regeneration of injured tissues ${ }^{22-25}$. Among the various types of stem cells, bone marrow-derived MSCs (BMSCs) are one of the most promising candidates for cell replacement therapy.

\section{Biological characteristics of MSCs}

MSCs are characterized with self-healing, selfrenewing, highly proliferative, differentiation potential, and adherent growing features. Despite expression of CD105, CD73 and CD90, these cells do not express CD45, CD34, CD14, CD19 and HLA-DR surface molecules. MSCs can be induced to differentiate toward bone cells, fat cells, cardiac muscle cells, nerve cells and epithelial cells transformation ${ }^{26,27}$. And the resulting cells must be characterized via evaluation of above mentioned markers.

\section{Growth factors to induce BMSCs differentiation for regeneration of inner ear cells}

In the presence of specific growth factors such as Sonic hedgehog and retinoic acid, BMSCs have the capacity to differentiate into sensory neurons ${ }^{27-29}$. In a recent study on BMSCs, the inner ear specific genes such as NF-M, neurog1, gluR4, neuroD, calretinin, neuN, tau, and GATA3 were up-regulated in the presence of bone morphogenetic protein 4 (BMP4), demonstrating their capacity to differentiate into auditory neuron-like cells in vitro $^{30}$. Rat BMSCs cultured in a medium containing glial cell-derived neurotrophic factor (GDNF), brain-derived neurotrophic factor (BDNF) and neurotrophin-3 (NT-3) for 14 days were differentiated into neuronal progenitor cells which in turn can differentiate into neuronal cells, and therefore might be useful for the treatment of sensorineural hearing loss (SNHL) ${ }^{31}$. It has also been reported that hMSCs differentiation into an intermediate neural progenitor stage is essential to obtain inner ear sensory lineages. In a study conducted by Alonso et al., neuron-like cells were obtained from neural progenitors grown in serum-free medium containing EGF and retinoic acid and then hair cell-like cells and auditory cells were generated from neuron-like cells ${ }^{32}$.

\section{Hearing recovery after transplantation of MSCs}

Deafness animal models have been used to transplant MSCs differentiated into sensory neuronlike cells ${ }^{27,33}$. Indeed a useful approach for inner ear supplementation with stem cells can be hMSCs systemic application ${ }^{34}$. For instance, the number of spiral ganglion neurons (SGNs) in guinea pig model is increased after transplantation of neural differentiated hMSCs resulting in mild hearing recovery after transplantation ${ }^{33}$.

The BMSCs have been fruitfully transplanted into young mice cochlea via the perilymphatic perfusion technique and further differentiated into fibrocyte-like (SLFlike) cells ${ }^{35}$. The hearing recovery after transplantation of MSCs into the inner ear cells may be occurred through two mechanisms. First, MSCs transdifferentiation into SLF-like cells may lead to compensate for lost SLFs, and second, transplanted MSCs may stimulate the host SLFs regeneration. Both mechanisms contribute to functional recovery of damaged SLF network and thus, transplantation of MSCs into the inner ear of rats with damaged SLFs significantly accelerates hearing recovery ${ }^{36}$. The potential of neural-induced human MSCs (NI-hMSCs) to help replace the lost cochlear cells in hearing loss mammals has been investigated. Indeed, grafted $\mathrm{NI}$-hMSCs migrate into the spiral ganglion where they expressed the neuron-specific marker, $\mathrm{NeuN}^{37}$. A study on transplantation of undifferentiated mouse BMSCs into normal and ouabain-treated gerbil cochleae and determination of their migratory patterns has demonstrated that survival of transplanted MSCs into the modiolus of the cochlea may result in regeneration of damaged $\mathrm{SGNs}^{38}$. In a similar study, rats 
with transplanted MSCs in the lateral wall demonstrated a significantly higher hearing recovery comparing to negative controls suggesting that MSCs transplantation results in hearing recovery through the repair of injured cochlear fibrocytes $^{39}$. Furthermore, bone marrow-derived cells may have the ability to attenuate cochlear injury by replacing or regenerating mesenchymal cells and fibrocytes in the inner ear ${ }^{40}$. These findings suggest that BMSCs can differentiate into neuronal progenitor cells and therefore may represent a promising biological element for treatment of inner ear disease, such as $\mathrm{SNHL}^{31}$. Furthermore, cells originating from bone marrow particularly those derived from hematopoietic stem cells (HSCs), seem to have the capibility to engraft into the inner ear ${ }^{41}$.

\section{Recombinant BMSCs}

Upon transplantation, BMSCs expressing recombinant IL-4 have the ability to remediate the inflammatory injury in autoimmune inner ear diseases ${ }^{42}$. In an attempt to provide a regenerative therapy for deafness in 2014, Buddy and co-workers successfully forced hBMSCs to express essential genes in the otic lineages ${ }^{43}$. Additionally, BDNF gene-modified MSCs are known to have a protective effect on the spiral ganglion cells ${ }^{44}$. Another example is genetic manipulation for Atoh1 which is a master gene for the differentiation of the hair cells. Indeed administration of Atoh1 expression can be highly beneficial for hair cell development and regeneration ${ }^{45}$. The neurosensory progenitors from bone marrow can also be converted to sensory hair cells through overexpression of Atoh- ${ }^{46}$. Moreover it is known that BMSCs can be induced to differentiate into hair cells through a combination of growth factor stimulation and expression of the transcription factor Atoh- $1^{46}$.

\section{Activation of BMSCs homing for hearing recovery}

In an attempt to determine the homing capacity of BMSCs, Tan and co-workers transplanted BMCs from green fluorescent protein (GFP) transgenic mice into the irradiated mice and then induced hearing injury by acoustic deafening. They observed GFP + cell migration to the deafened cochlea particularly at the perilymphatic compartment walls, spiral ligament and limbus regions ${ }^{47}$. A new promising strategy for the activation of stem cell homing factors is induction of stem cell homing factors (SDF-1 and MCP-1) in the host cochlear tissue, and their receptors (CCR2 and CXCR4) in transplanted $\mathrm{MSCs}^{48}$. This knowledge suggests that activation of stem cell homing can be an effective strategy for hearing recovery.

\section{Adipose tissue derived MSCs}

Adipose-derived stem cells (ASCs) can be isolated easily from blood vessels of adipose tissue ${ }^{49}$. These multipotent cells reside in the stromal vascular fraction and exhibit great plasticity and multilineage differentiation potential ${ }^{50}$. ASCs are capable to differentiate into all mesodermal lineages such as adipose tissue and other connective tissues (bone, cartilage, and muscles) ${ }^{51}$. However there are evidences that ASCs can be helpful in hearing repair. An original proof for developments of ASC-based treatments for deafness is that ASCs can migrate to the tissue damage location and express trophic factors ${ }^{52}$. Systemic infusion of ASC has been reported to significantly protect hair cells and improve hearing function in guinea pig with autoimmune hearing loss ${ }^{52}$.

Additionally, the paracrine activity of hASC is known to help hearing restoration. The hASCs cause decrease in proliferation of antigen-specific Th1/Th17 cells by inducing the production of anti-inflammatory cytokine interleukin-10 in splenocytes. They also induce the generation of antigen-specific CD4+ CD25+ Foxp3+ Treg cells with the capacity to suppress autoantigenspecific T-cell responses ${ }^{53}$. In a study on guinea pig, the capability of ASCs to differentiate into neuron-like cells was investigated representing ASCs obtained from the neck as a good cell source for autologous cellbased regenerative methods ${ }^{54}$. ASCs are able to migrate to the location of tissue damage and express trophic factors throughout intracochlear implantation in guinea pig model of acoustic trauma ${ }^{52}$.

A strategy based on combination of total protein extract transfer from VOT-E36 otic epithelial cells and Atoh1 overexpression represents a novel method to convert ASCs into hair cell-like cells. Atoh1 overexpression successfully transforms VOT-E36 cells into hair celllike cells which further can attract contacts from spiral ganglion neurons in a co-culture medum ${ }^{55}$. The above mentioned evidences suggest that ASCs may be suitable tools to be used in regenerative medicine, because of their high plasticity and trophic features. Combined with conventional therapies, such as cochlear implantation, the feasibility of local ASCs delivery can contribute to future innovative biological strategies to enhance the endogenous reparative processes in the early stage of injury.

\section{Umbilical Cord MSCs (UCMSCs)}

Abundance of umbilical cords, low immune rejection and nontumorigenic properties, have made UCMSCs to be considered an excellent source for cell transplantation therapies and regenerative medicine ${ }^{56}$. It is known that the expression of Atoh1 leads to induce the differentiation of hUCMSCs into cells that are morphologically and immunocytochemical similar to inner ear hair cells ${ }^{57}$.

Interestingly, there are evidences that UCMSCs are beneficial even without genetic manipulation. For instance, intravenous transplantation of intact UCMSCs can itself improve hearing thresholds via relocation and increasing in the number of spiral ganglion neurons (SGNs) ${ }^{58}$. Furthermore, other findings indicate that the migration of transplanted human cord blood CD133+ hematopoietic stem cells (HSC) to the inner ear leads to the resumption of deafened cochlea of oto-injured mice ${ }^{59}$. The implanted cells are further integrated into the cochlea of the inner ear suggesting a possible strategy for rehabilitation of inner ear. In addition, the number of spiral ganglion cells and outer hair cells are increased and 
according to auditory brainstem response (ABR) tests, the hearing level improved ${ }^{60}$.

\section{Olfactory stem cells (mesenchymal-like stem cells)}

The nasal olfactory stem cells from the human olfactory mucosa can be efficiently used for autologous stem cell-based therapies mainly due to their high abundance and easy accessibility ${ }^{61}$. Olfactory stem cells have been successfully injected into the cochlea of mice with excellent outcomes. The transplantation of adult human olfactory mucosa-derived stem cells has been also found to help preserve auditory function during earlyonset progressive sensorineural hearing loss ${ }^{62}$. There are also evidences that the human mesenchymal-like stem cells derived from nasal tissue can repair spiral ganglion loss in experimentally injured cochlear of neonatal rats ${ }^{63}$.

\section{DISCUSSION}

Due to highly promising nature of stem cells for regenerative medicine, intensive researches are being conducted to treat various diseases using embryonic, adult or induced pluripotent stem cells ${ }^{64}$. Cell based researches include a wide range of attempts such as treatments to aid nerve protection ${ }^{65,66}$ and regeneration or recovering cardiac tissue ${ }^{67}$. In this regard, various aspects of developmental biology and molecular signaling networks must be considered ${ }^{68,69}$. Multi differentiation potential of MSCs and their capability to migrate into acute injury location make these cells suitable candidate for gene and cell therapy ${ }^{70-74}$. MSCs have been demonstrated to be helpful in treating inner ear inflammatory damage because they exhibit multidirectional differentiation potential, immunosuppressive function and low immunogenicity ${ }^{75}$. Among MSCs, BMSCs have been widely studied and are comparably more practical ${ }^{58}$. Various studies indicate potential of BMSC to deliver therapeutic molecules and restore cochlear cells. BMSC transplantation could be utilized through three different strategies for inner ear treatment; restoration of missing cells, providing growth factors and delivering genes. Current findings on MSCs treatment efficacy in otorhinolaryngology are based on animal models. The true impact on clinical treatment will not be revealed until clinical trials confirm functional outcomes in human medicine. Two limitations of MSCs replacement is the process of MSCs relocation into the inner ear and the fact that stem cells do not spontaneously divide to replace damaged sensory cells. In spite of high plasticity, the capability of trans-differentiation along nonmesodermal lineages is highly questionable. Particularly, possible neural trans-differentiation of MSCs, such as ASCs, is still being challenged ${ }^{76-78}$. Studies have confirmed the ability of the easily obtained and autologously available adult human stem cell to repair auditory neuron loss. However research should progress toward the establishment of cell-based therapies for certain forms of deafness ${ }^{63}$. In vitro studies have shown the differentiation of mouse MSCs into glutamatergic sensory neurons ${ }^{28}$ and $\mathrm{HC}$-like cells ${ }^{46,79}$. Since bone marrow transplants have been used by clinicians for decades, it would be more likely to move from animal trials using hMSCs, and hematopoietic stem cells toward clinical trials ${ }^{67}$.

\section{CONCLUSION}

Stem cell therapy for sensorineural hearing aims to repair the hair cells and spiral ganglion cells to improve the auditory function. MSC transplantation for the treatment of hearing loss has opened up a new way of thinking. This review suggests that BMSCs are more capable to migrate and survive into the cochlear tissues which them suitable to be used in transplantation as a strategy for regenerating inner ear and treatment of SNHL.

\section{REFERENCES}

1. Li $\mathrm{H}$, Liu $\mathrm{H}$, Heller S. Pluripotent stem cells from the adult mouse inner ear. Nat Med. 2003;9(10):1293-9

2. Oshima K, Grimm CM, Corrales CE, Senn P, Monedero RM, Géléoc GS, et al. Differential distribution of stem cells in the auditory and vestibular organs of the inner ear. $\mathrm{J}$ Assoc Res Otolaryngol. 2007;8(1):18-31.

3. Groves AK. The challenge of hair cell regeneration. Exp Biol Med. 2010;235(4):434-46

4. Jongkamonwiwat N, Zine A, N Rivolta M. Stem cell based therapy in the inner ear: Appropriate donor cell types and routes for transplantation. Curr Drug Targets. 2010;11(7):888-97.

5. De Felipe M, Feijoo Redondo A, Garcia-Sancho J, Schimmang T, Alonso M. Cell-and gene-therapy approaches to inner ear repair. Histol Histopathol. 2011;26(7):923-40.

6. Li H, Roblin G, Liu H, Heller S. Generation of hair cells by stepwise differentiation of embryonic stem cells. Proc Natl Acad Sci USA. 2003;100(23):13495-500.

7. Oshima K, Shin K, Diensthuber M, Peng AW, Ricci AJ, Heller S. Mechanosensitive hair cell-like cells from embryonic and induced pluripotent stem cells. Cell. 2010;141(4):704-16.

8. Corrales CE, Pan L, Li H, Liberman MC, Heller S, Edge AS. Engraftment and differentiation of embryonic stem cell-derived neural progenitor cells in the cochlear nerve trunk: Growth of processes into the organ of corti. J Neurobiol. 2006;66(13):1489-500.

9. Altschuler RA, O'Shea KS, Miller JM. Stem cell transplantation for auditory nerve replacement. Hear Res. 2008;242(1):110-6.

10.Shi F, Corrales CE, Liberman MC, Edge AS. BMP4 induction of sensory neurons from human embryonic stem cells and reinnervation of sensory epithelium. Eur J Neurosci. 2007;26(11):3016-23.

11. Reyes JH, O'Shea KS, Wys NL, Velkey JM, Prieskorn DM, Wesolowsk $\mathrm{K}$, et al. Glutamatergic neuronal differentiation of mouse embryonic stem cells after transient expression of neurogenin 1 and treatment with BDNF and GDNF: In vitro and in vivo studies. J Neurosci. 2008;28(48):12622-31.

12.Erdö F, Bührle C, Blunk J, Hoehn M, Xia Y, Fleischmann B, et al. Hostdependent tumorigenesis of embryonic stem cell transplantation in experimental stroke. J Cereb Blood Flow Metab. 2003;23(7):780-5.

13. Sell S. Stem cell origin of cancer and differentiation therapy. Crit Rev Oncol Hematol. 2004;51(1):1-28.

14.Pauley S, Kopecky B, Beisel K, Soukup G, Fritzsch B. Stem cells and molecular strategies to restore hearing. Panminerva Med. 2008;50(1):41.

15. Hu Z, Corwin JT. Inner ear hair cells produced in vitro by a mesenchymal-to-epithelial transition. Proc Natl Acad Sci USA. 2007;104(42):16675-80.

16. Hermann A, Gastl R, Liebau S, Popa MO, Fiedler J, Boehm BO, et 
al. Efficient generation of neural stem cell-like cells from adult human bone marrow stromal cells. J Cell Sci. 2004;117(19):4411-22.

17. Krabbe C, Zimmer J, Meyer M. Neural transdifferentiation of mesenchymal stem cells- A critical review. APMIS. 2005;113(1112):831-44

18. Caddick J, Kingham PJ, Gardiner NJ, Wiberg M, Terenghi G. Phenotypic and functional characteristics of mesenchymal stem cells differentiated along a Schwann cell lineage. Glia. 2006;54(8):840-9.

19. Yang Q, Mu J, Li Q, Li A, Zeng Z, Yang J, et al. A simple and efficient method for deriving neurospheres from bone marrow stromal cells. Biochem Biophys Res Commun. 2008;372(4):520-4.

20. Radtke C, Schmitz B, Spies M, Kocsis J, Vogt P. Peripheral glial cell differentiation from neurospheres derived from adipose mesenchymal stem cells. Int J Dev Neurosci. 2009;27(8):817-23.

21. Kim SS, Yoo SW, Park TS, Ahn SC, Jeong HS, Kim JW, et al. Neural induction with neurogenin1 increases the therapeutic effects of mesenchymal stem cells in the ischemic brain. Stem Cells. 2008;26(9):2217-28.

22. Kim J, Hematti P. Mesenchymal stem cell-educated macrophages: A novel type of alternatively activated macrophages. Exp hematol. 2009;37(12):1445-53.

23.Phinney DG, Prockop DJ. Concise review: Mesenchymal stem/ multipotent stromal cells: The state of transdifferentiation and modes of tissue repair-current views. Stem cells. 2007;25(11):2896-902.

24.King SN, Hanson SE, Hematti P, Thibeault SL. Current applications of mesenchymal stem cells for tissue replacement in otolaryngologyhead and neck surgery. Am J Stem Cells. 2012;1(3):225.

25.Prockop DJ, Oh JY. Mesenchymal stem/stromal cells (MSCs): Role as guardians of inflammation. Mol Ther. 2012;20(1):14-20.

26. De Girolamo L, Lucarelli E, Alessandri G, Antonietta Avanzini M, Ester Bernardo M, Biagi E, et al. Mesenchymal stem/stromal cells: A new"cells as drugs"paradigm. Efficacy and critical aspects in cell therapy. Curr Pharm Des. 2013;19(13):2459-73.

27. Kondo T, Matsuoka AJ, Shimomura A, Koehler KR, Chan RJ, Miller $\mathrm{JM}$, et al. Wht signaling promotes neuronal differentiation from mesenchymal stem cells through activation of T/x3. Stem cells. 2011;29(5):836-46.

28. Kondo T, Johnson SA, Yoder MC, Romand R, Hashino E. Sonic hedgehog and retinoic acid synergistically promote sensory fate specification from bone marrow-derived pluripotent stem cells. Proc Natl Acad Sci USA. 2005;102(13):4789-94.

29. Yu Z, Wu S, Liu Z, Lin H, Chen L, Yuan X, et al. Sonic hedgehog and retinoic acid induce bone marrow-derived stem cells to differentiate into glutamatergic neural cells. J Immunoassay Immunochem. 2015;36(1):1-15

30.Peng T, Zhu G, Dong Y, Zeng J, Li W, Guo W, et al. BMP4: A possible key factor in differentiation of auditory neuron-like cells from bonederived mesenchymal stromal cells. Clin Lab. 2015;61:1171-8.

31.Lee JH, Kang WK, Seo JH, Choi MY, Lee YH, Kim HM, et al. Neural differentiation of bone marrow-derived mesenchymal stem cells: Applicability for inner ear therapy. Korean J Audiol. 2012;16(2):47-53.

32.Durán Alonso MB, Feijoo-Redondo A, Conde de Felipe M, Carnicero E, García AS, García-Sancho J, et al. Generation of inner ear sensory cells from bone marrow-derived human mesenchymal stem cells. Regen Med. 2012;7(6):769-83.

33. Cho YB, Cho HH, Jang S, Jeong HS, Park JS. Transplantation of neural differentiated human mesenchymal stem cells into the cochlea of an auditory-neuropathy guinea pig model. J Korean Med Sci. 2011;26(4):492-8.

34. Choi BY, Song JJ, Chang SO, Kim SU, Oh SH. Intravenous administration of human mesenchymal stem cells after noiseor drug-induced hearing loss in rats. Acta oto-laryngologica. 2012;132(sup1):S94-102.
35. Kasagi $\mathrm{H}$, Kuhara T, Okada $\mathrm{H}$, Sueyoshi N, Kurihara $\mathrm{H}$. Mesenchymal stem cell transplantation to the mouse cochlea as a treatment for childhood sensorineural hearing loss. Int J Pediatr Otorhinolaryngol. 2013;77(6):936-42.

36.Sun Gw, Fujii M, Matsunaga T. Functional interaction between mesenchymal stem cells and spiral ligament fibrocytes. J Neurosc Res. 2012;90(9):1713-22.

37.Jang S, Cho HH, Kim SH, Lee KH, Jun JY, Park JS, et al. Neuralinduced human mesenchymal stem cells promote cochlear cell regeneration in deaf Guinea pigs. Clin Exp Otorhinolaryngol. 2015;8(2):83.

38. Matsuoka AJ, Kondo T, Miyamoto RT, Hashino E. Enhanced Survival of Bone-Marrow-Derived Pluripotent Stem Cells in an Animal Model of Auditory Neuropathy. Laryngoscope. 2007;117(9):1629-35.

39. Kamiya K, Fujinami Y, Hoya N, Okamoto Y, Kouike H, Komatsuzak $\mathrm{R}$, et al. Mesenchymal stem cell transplantation accelerates hearing recovery through the repair of injured cochlear fibrocytes. Am J Pathol. 2007;171(1):214-26.

40.Lang $\mathrm{H}$, Ebihara $\mathrm{Y}$, Schmiedt RA, Minamiguchi H, Zhou D, Smythe N et al. Contribution of bone marrow hematopoietic stem cells to adult mouse inner ear: mesenchymal cells and fibrocytes. J Comp Neurol. 2006;496(2):187-201.

41.Domen J, Wagers A, Weissman IL. Bone marrow (hematopoietic) stem cells. Regen Med. 2006:13.

42.Tan CQ, Gao X, Guo L, Huang H. Exogenous IL-4-expressing bone marrow mesenchymal stem cells for the treatment of autoimmune sensorineural hearing loss in a guinea pig model. BioMed Res Int. 2014;2014.

43.Boddy SL, Chen W, Romero-Guevara R, Kottam L, Bellantuono I, Rivolta MN. Inner ear progenitor cells can be generated in vitro from human bone marrow mesenchymal stem cells. Regen Med. 2012;7(6):757-67.

44.Nayagam BA, Muniak MA, Ryugo DK. The spiral ganglion: Connecting the peripheral and central auditory systems. Hear Res. 2011;278(1):2-20.

45. Richardson RT, Atkinson PJ. Atoh1 gene therapy in the cochlea for hair cell regeneration. Expert Opin Biol Ther. 2015;15(3):417-30.

46.Jeon SJ, Oshima K, Heller S, Edge AS. Bone marrow mesenchymal stem cells are progenitors in vitro for inner ear hair cells. Mol Cell Neurosci. 2007;34(1):59-68.

47. Tan BTG, Lee MMG, Ruan R. Bone marrow-derived cells that home to acoustic deafened cochlea preserved their hematopoietic identity. J Comp Neurol. 2008;509(2):167-79.

48. Kamiya K. Inner ear cell therapy targeting hereditary deafness by activation of stem cell homing factors. Front Pharmacol. 2015;6:2.

49. Rodriguez AM, Elabd C, Amri EZ, Ailhaud G, Dani C. The human adipose tissue is a source of multipotent stem cells. Biochimie. 2005;87(1):125-8.

50.Bourin P, Bunnell BA, Casteilla L, Dominici M, Katz AJ, March KL, et al. Stromal cells from the adipose tissue-derived stromal vascular fraction and culture expanded adipose tissue-derived stromal/stem cells: A joint statement of the International Federation for Adipose Therapeutics and Science (IFATS) and the International Society for Cellular Therapy (ISCT). Cytotherapy. 2013;15(6):641-48.

51.Saulnier N, Puglisi MA, Lattanzi W, Castellini L, Pani G, Leone G, et al. Gene profiling of bone marrow-and adipose tissue-derived stromal cells: A key role of Kruppel-like factor 4 in cell fate regulation. Cytotherapy. 2011;13(3):329-40.

52. Fetoni AR, Lattanzi W, Eramo SLM, Barba M, Paciello F, Moriconi C, et al. Grafting and early expression of growth factors from adiposederived stem cells transplanted into the cochlea, in a guinea pig model of acoustic trauma. Front Cell Neurosci. 2014;8.

53.Zhou Y, Yuan J, Zhou B, Lee AJ, Lee AJ, Ghawji Jr M, et al. The 
therapeutic efficacy of human adipose tissue-derived mesenchymal stem cells on experimental autoimmune hearing loss in mice. Immunology. 2011;133(1):133-40.

54.Frölich K, Scherzed A, Mlynski R, Technau A, Hagen R, Kleinsasser $\mathrm{N}$, et al. Multipotent stromal cells for autologous cell therapy approaches in the guinea pig model. ORL. 2011;73(1):9-16.

55.Lin Z, Perez P, Sun Z, Liu JJ, Shin JH, Hyrc KL, et al. Reprogramming of single-cell derived mesenchymal stem cells into hair cell-like cells. Otol Neurotol. 2012;33(9):1648-55.

56. Fong CY, Richards M, Manasi N, Biswas A, Bongso A. Comparative growth behaviour and characterization of stem cells from human Wharton's jelly. Reprod Biomed Online. 2007;15(6):708-18.

57. Devarajan K, Forrest ML, Detamore MS, Staecker H. Adenovectormediated gene delivery to human umbilical cord mesenchymal stromal cells induces inner ear cell phenotype. Cellular Reprogramming (Formerly" Cloning and Stem Cells"). 2013;15(1):4354.

58. Choi MY, Yeo SW, Park KH. Hearing restoration in a deaf animal model with intravenous transplantation of mesenchymal stem cells derived from human umbilical cord blood. Biochem Biophys Res Commun. 2012;427(3):629-36.

59. Revoltella RP, Papini S, Rosellini A, Michelini M, Franceschini V, Ciorba $A$, et al. Cochlear repair by transplantation of human cord blood CD133+ cells to nod-scid mice made deaf with kanamycin and noise. Cell transplant. 2008;17(6):665-78.

60. Burra P, Arcidiacono D, Bizzaro D, Chioato T, Di Liddo R, Banerjee A, et al. Systemic administration of a novel human umbilical cord mesenchymal stem cells population accelerates the resolution of acute liver injury. BMC Gastroenterol. 2012;12(1):88.

61. Stamegna JC, Girard SD, Veron A, Sicard G, Khrestchatisky M, Feron $F$, et al. A unique method for the isolation of nasal olfactory stem cells in living rats. Stem Cell Res. 2014;12(3):673-9.

62.Pandit SR, Sullivan JM, Egger V, Borecki AA, Oleskevich S. Functional Effects of Adult Human Olfactory Stem Cells on EarlyOnset Sensorineural Hearing Loss. Stem Cells. 2011;29(4):670-7.

63.Bas E, Van De Water TR, Lumbreras V, Rajguru S, Goss G, Hare $\mathrm{JM}$, et al. Adult human nasal mesenchymal-like stem cells restore cochlear spiral ganglion neurons after experimental lesion. Stem Cells Dev. 2013;23(5):502-14.

64.Ghasemi-Dehkordi P, Allahbakhshian-Farsani M, Abdian N, Mirzaeian $A$, Saffari-Chaleshtori J, Heybati F, et al. Comparison between the cultures of human induced pluripotent stem cells (hiPSCs) on feederand serum-free system (Matrigel matrix), MEF and HDF feeder cell lines. J Cell Commun Signal. 2015;9(3):233-46.

65. Jami MS, Salehi-Najafabadi Z, Ahmadinejad F, Hoedt E, Chaleshtori $\mathrm{MH}$, Ghatrehsamani M, et al. Edaravone leads to proteome changes indicative of neuronal cell protection in response to oxidative stress. Neurochem Int. 2015;90:134-41.
66. Jami MS, Pal R, Hoedt E, Neubert TA, Larsen JP, Møller SG. Proteome analysis reveals roles of L-DOPA in response to oxidative stress in neurons. BMC Neurosci. 2014;15(1):93.

67. Trounson A, Thakar RG, Lomax G, Gibbons D. Clinical trials for stem cell therapies. BMC Med. 2011;9(1):52.

68. Mahmoudian-sani MR, Mehri-Ghahfarrokhi A, Ahmadinejad F, Hashemzadeh-Chaleshtori M, Saidijam M, Jami MS. MicroRNAs: Effective elements in ear-related diseases and hearing loss. Eur Arch Otorhinolaryngol. 2017:1-8.

69.Mahmoodian SMR, Hashemzadeh-Chaleshtori M, MehriGhahfarrokhi A, Ghasemi-Dehkordi P, Saidijam M, Jami MS. MicroRNA-183 Family in Inner Ear: Hair Cell Development and Deafness. J Audiol Otol. 2016;20(3):131-8.

70.Xiao Y, Wang Y, Li L, Li Y, Pang Y, Song J, et al. Homing of chloromethylbenzoyl ammonia-labeled bone marrow mesenchymal stem cells in an immune-mediated bone marrow failure mouse model in vivo. Genet Mol Res. 2014;13(1):11-21.

71.Fawzy SA, Abo-Elnou RKE-d, El Dfa EM. The possible role of mesenchymal stem cells therapy in the repair of experimentally induced colitis in male albino rats. Int J Stem Cells. 2013;6(2):92103.

72.Liu N, Tian J, Cheng J, Zhang J. Migration of CXCR4 gene-modified bone marrow-derived mesenchymal stem cells to the acute injured kidney. J Cell Biochem. 2013;114(12):2677-89.

73. Yang D, Sun S, Wang Z, Zhu P, Yang Z, Zhang B. Stromal cellderived factor-1 receptor CXCR4-overexpressing bone marrow mesenchymal stem cells accelerate wound healing by migrating into skin injury areas. Cell Reprogram. 2013;15(3):206-15.

74.Gao Z, Cheng P, Xue Y, Liu Y. Vascular endothelial growth factor participates in modulating the C6 glioma-induced migration of rat bone marrow-derived mesenchymal stem cells and upregulates their vascular cell adhesion molecule-1 expression. Exp Ther Med. 2012;4(6):993-8.

75.Sharif S, Nakagawa T, Ohno T, Matsumoto M, Kita T, Riazuddin S, et al. The potential use of bone marrow stromal cells for cochlear cell therapy. Neuroreport. 2007;18(4):351-4.

76. Hu Y, Tang X, He H. Gene expression during induced differentiation of sheep bone marrow mesenchymal stem cells into osteoblasts. Genet Mol Res. 2013;12(4):6527-34.

77. Heng BC, Saxena P, Fussenegger M. Heterogeneity of baseline neural marker expression by undifferentiated mesenchymal stem cells may be correlated to donor age. J Biotechnol. 2014;174:29-33.

78. Yang J, Xiong L, Wang R, Sun JS, Hirche C. Adipose-derived stem cells from the breast. J Res Med Sci. 2014;19(2).

79. Qin H, Zhao LD, Sun JH, Ren LL, Guo WW, Liu HZ, et al. The differentiation of mesenchymal stem cells into inner ear hair cell-like cells in vitro. Acta oto-laryngologica. 2011;131(11):1136-41. 